\title{
Attitudes of Patients with Multiple Sclerosis Towards Disease and Physical Activity Behaviors During the COVID-19 Pandemic
}

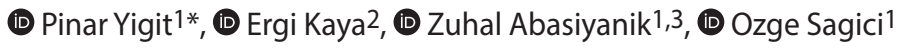 \\ ${ }^{1}$ Dokuz Eylul University, Graduate School of Health Science, Izmir, Turkey \\ 2Dokuz Eylul University Faculty of Medicine, Department of Neurology, Izmir, Turkey \\ 3Izmir Katip Celebi University Faculty of Health Sciences, Department of Physiotherapy and Rehabilitation, Izmir, Turkey
}

\section{Abstract}

Objective: This study aimed to investigate the considerations of patients with multiple sclerosis (pwMS) related to their disease and treatment, the frequency of going out, treatment and routine examination interruptions, and physical exercise habits during the pandemic.

Materials and Methods: Seven hundred forty-four pwMS (mean age: 41.44 years, 29.2\% male, 70.8\% female), who were followed in MS Outpatient Clinic in Dokuz Eylul University Hospital, Izmir, Turkey, were recruited. A structured survey was created and administered via phone to assess attitudes towards disease, the frequency of going out, treatment and routine examination interruptions, and physical exercise behavior. Additionally, demographic and clinical characteristics, their health status, and coronavirus disease 2019 (COVID-19) symptoms were also asked.

Results: $66.5 \%(n=495)$ of responders stated that their thoughts about the disease did not change during the pandemic. 94\% ( $n=699)$ of the pwMS reported that they did not experience any disruption in their treatment. 59.9\% ( $n=446)$ of the pwMS indicated no disruption in their routine controls. $25 \%$ of responders reported less physical activity, $17.1 \%$ reported more physical activity, $16.5 \%$ continued physical activity as usual. $41.4 \%$ reported that they did not perform any physical activity in the past and did not do so during the pandemic.

Conclusion: This large cross-sectional study has shown that the attitudes of the majority of pwMS towards their disease have not changed. The continuation of the follow-up of the patients during the COVID-19 period with telehealth applications may ensure the maintenance of treatment adherence and patients' attitudes about the disease.

Keywords: Multiple sclerosis, COVID-19 pandemic, clinical practice, physical activity, exercise, treatment adherence

\section{Introduction}

The global outbreak of the coronavirus disease 2019 (COVID-19) has dramatically spread from person to person worldwide, and its dissemination is still intensifying in many areas. The COVID-19 represents various clinical manifestations, including fever, dry cough, myalgia, upper respiratory tract, gastrointestinal symptoms, and fatigue (1).

Multiple sclerosis (MS) is the immune-mediated chronic, inflammatory disease of the central nervous system, and pwMS receive disease-modifying therapies. Those with preexisting medical conditions, additional comorbidities, and advanced age have the infection-related potential risks factors for COVID-19 $(2,3)$. In managing MS disease, there are many care services such as routine clinical examinations, relapse management, and rehabilitation services that will require patients to access the hospital. As mentioned above, home confinement has caused pwMS to postpone to schedule their follow-up examinations, the laboratory blood tests, their magnetic resonance, initiation therapies, or taking pulse methyl-prednisolone treatment (4).

Evidence is accumulating that MS or MS immunotherapies do not increase the incidence of infection or the severity of infection (5). However, in the first months of the pandemic outbreak, there was no experience in this regard. This study aimed to collect data on the considerations of patients with MS (pwMS) related to their disease and treatment and daily routine outside and physical exercise habits during the pandemic. In addition, we both followed their ongoing therapies in

Address for Correspondence: Pinar Yigit, Dokuz Eylul University, Graduate School of Health Science, Izmir, Turkey E-mail: psypinaryigit@gmail.com ORCID-ID: orcid.org/0000-0002-1461-069X

Received: 07.01.2022 Accepted: 25.01.2022

${ }^{\circ}$ Copyright 2021 by the Journal of Multiple Sclerosis Research published by Galenos Publishing House. 
the current situation and interrogated the impact of home confinement in pwMS.

\section{Materials and Methods}

\section{Participants and Procedure}

This study protocol was confirmed by the Turkey Ministry of Health and Dokuz Eylul University Ethics Committee (protocol number: 5507-GOA and approval number: 2020/15-32). Informed consent was obtained verbally from all participants before the assessment during the pandemic.

The inclusion criteria were the diagnosis of definite MS according to revised criteria by an MS neurologist confirmation (6), and being aged 18 years and older. The exclusion criteria were having a current neurological disease other than MS, having any verbal skill deficiency or impairment that might influence response to the survey.

During the recruitment period, the routine assessments were performed via teleassessment by neurologist. Patients could reach the MS nurses, psychologists, and physiotherapists for their questions.

\section{Assessments}

We asked about the demographic characteristics (age, education level, marital status, residence, employment status, number of households, and children). Besides, we also inquired about their health status by using the survey mentioned above, involving COVID-19 symptoms for them or their close relatives, the last time of hospital admission for various reasons, the last time they went out, and the level of physical activity. We obtained clinical features from our database (disease duration, course of MS, disability level, and using disease-modifying therapies (DMTs), Expanded Disability Status Scale (EDSS).

A structured survey was created by the three members of our research group, including a psychologist, physiotherapist, and senior neurologist. The survey was performed by the Multiple Sclerosis Research Group, which included an MS neurologist specialized in managing pwMS, physiotherapists, psychologists, and MS nurses. Seven hundred forty-four (female 70.8\%, 29.2\% male) respondents were reached by mobile phones from May 5 to June 5, 2020, in the MS Outpatient Clinic of a tertiary hospital affiliated with the Medical Sciences of Dokuz Eylul University, Izmir, Turkey. We enrolled with patient self-reporting and questionnaire, including information about MS and COVID-19, via phone calls. Firstly, we interviewed and got feedback from $10 \%$ of participants in this study and ensured survey questions to be consistent. We phoned again to pwMS, who did not respond to the first call, about 5 or 7 days later. The content is provided in the tables presented in the results section.

\section{Statistical Analysis}

Statistical analyses were performed with IBM ${ }^{\otimes}$ SPSS ${ }^{\circledR}$ Statistics for Windows (Version 25.0. Armonk, NY: IBM Corp.). Descriptive analysis was shown as means and standard deviation (SD) for numerical variables and percentages for categorical variables. The t-tests analysis and chi-square test were performed to obtain two group comparisons between the individual groups. The Pearson correlation coefficient $r$ was used to examine the relationship between the numerical variables. The One-way ANOVA was used to compare EDSS among the groups (positive, negative, neutral/not changed, the decreased frequency, never get out). The Levene test was employed to assess the homogeneity of the variances. A $p$ value $\leq 0.05$ was considered statistically significant.

\section{Results}

Seven hundred forty-four pwMS were interviewed by phone during the first six-month-period of COVID-19 pandemic. 527 (70.8\%) and 217 (29.2\%) were female and male, respectively. The mean age, EDSS, and disease duration were 41.44 \pm 11.58 years (range: between 18 and 77 years), $2.12 \pm 2.24$ (range: between 0 and 8.5), and $11.54 \pm 8.5$ years (range: between 0 and 41), respectively (Table 1). Also, thirty-one cases who were the relatives of pwMS (4.1\%) had reported as COVID-19 positive. None of the pwMS experienced new relapse/attacks from the initial of pandemic to the date when we administered the survey.

There were 608 (81.7\%), 94 (12.6\%), 23 (3.1\%), and 19 (2.6\%) patients with relapsing-remitting course (RRMS), secondaryprogressive (SPMS), primary-progressive (PPMS), and clinically isolated syndrome (CIS), respectively. Thirty-one (4.1\%) patients were not administered any DMTs. Nine patients (1.2\%) had systemic corticosteroids, 130 (17.5\%) interferon-beta, 88 (11.8\%) glatiramer acetate, 218 (39.2\%) fingolimod, 14 (1.9\%) dimethyl fumarate, 43 (5.8\%) teriflunomide, 28 (3.8\%) natalizumab, 176 (23.7\%) ocrelizumab, 4 (0.5\%) azathioprine, and 1 (0.1\%) rituximab. One patient $(0.1 \%)$ was in the phase 3 trial of ofatumumab (Table 2).

Responders were asked, "Have your attitude changed about MS during the pandemic?". Four hundred and ninety-five pwMS (66.5\%) answered as "not changed" regarding their thoughts about the disease during the pandemic, while 249 pwMS (33.5\%) responded as "changed". Age, duration of the formal education, marital status, disease duration, EDSS, and last time they went out were not significantly different between those with changing and fixed thoughts about MS ( $p>0.05)$. Those who did not change their attitudes on their diseases generally stated that their diseases were also under control during the pandemic. Of the participants whose attitudes had changed, $158(21.2 \%)$ rated it as "If I get infected with the virus, MS symptoms will get worse" (Table 3 ).

One hundred fourteen (15.3\%) responders stated that the frequency of going out did "not changed" during the pandemic, 501 (67.3) rated as "decreased", and 129 (17.3) rated as "never 


\begin{tabular}{|c|c|}
\hline & Mean (SD) \\
\hline Age (years) & $41.44 \pm 11.58$ \\
\hline \multicolumn{2}{|l|}{ Gender, n (\%) } \\
\hline Female & $527(70.8 \%)$ \\
\hline Male & $217(29.2 \%)$ \\
\hline \multicolumn{2}{|l|}{ Marital status, n (\%) } \\
\hline Married & $508(68.2)$ \\
\hline Single & $236(32.0)$ \\
\hline Number of households & $2.13 \pm 2.00$ \\
\hline Number of children & $1.11 \pm 0.97$ \\
\hline \multicolumn{2}{|l|}{ Educational status, n (\%) } \\
\hline Primary school & $130(17.5)$ \\
\hline Secondary school & $60(8.1)$ \\
\hline High school & $199(26.7)$ \\
\hline University & $342(46.0)$ \\
\hline \multicolumn{2}{|l|}{ Marital status } \\
\hline Married & $506(68.0)$ \\
\hline Single & $234(31.5)$ \\
\hline \multicolumn{2}{|l|}{ Disease course, n (\%) } \\
\hline Relapsing-remitting MS & $608(81.7 \%)$ \\
\hline Secondary progressive MS & $94(12.6 \%)$ \\
\hline Primary progressive MS & $23(3.1 \%)$ \\
\hline Clinically isolated MS & $19(2.6 \%)$ \\
\hline $\mathrm{EDSS}^{* * *}$ & $2.17 \pm 2.28$ \\
\hline Disease duration (years) & $11.55 \pm 8.50$ \\
\hline \multicolumn{2}{|c|}{$\begin{array}{l}\text { EDSS: Expanded Disability Status Scale, MS: Multiple sclerosis, SD: Standarc } \\
\text { deviation }\end{array}$} \\
\hline
\end{tabular}

get out". The mean EDSS of those rated "never get out" was significantly higher than other groups. Two hundred forty-nine pwMS (33.5\%) reported that staying at home negatively affected their disease, while 114 participants (15.3\%) reported that it had a positive effect and 381 (51.2\%) had no effect (Table 4).

Six hundred ninety-nine pwMS (94.0\%) reported that they had not experienced an interruption in the treatment due to the pandemic. Twenty-five pwMS (3.4\%) stated that they could not get planned monthly methylprednisolone because they could not go to the hospital during the pandemic. $0.4 \%$ of patients quit the treatment because of the fear of getting COVID-19 (Table 5).

Four hundred forty-six pwMS (59.9\%) reported no interruption in their routine controls. Two hundred forty-two respondents (32.5\%) stated that their routine examination had been performed viaa telephone call by a neurologist. The examinations of 56 respondents (7.4\%) had not been completed for several

\begin{tabular}{|l|l|}
\hline \multicolumn{2}{|l|}{ Table 2. Data regarding the medications used for MS } \\
\hline Fingolimod & $\mathrm{n}(\%)$ \\
\hline Ocrelizumab & $218(39.2)$ \\
\hline Interferon-beta & $176(23.7)$ \\
\hline Glatiramer acetate & $130(17.5)$ \\
\hline Natalizumab & $88(11.8)$ \\
\hline Dimethyl fumarate & $28(3.8)$ \\
\hline Azathioprine & $14(1.9)$ \\
\hline Rituximab & $4(0.5)$ \\
\hline Ofatumumab phase 3 study & $1(0.1)$ \\
\hline Monthly pulse methylprednisolone & $1(0.1)$ \\
\hline No treatment & $9(1.2)$ \\
\hline
\end{tabular}

MS: Multiple sclerosis

Table 3. Has your attitude changed about MS during the pandemic?

\begin{tabular}{|c|c|c|c|}
\hline & Given answers & n (\%) & EDSS \\
\hline $\begin{array}{l}\text { Not changed } \\
(66.5 \%)\end{array}$ & $\begin{array}{l}\text { It has not } \\
\text { changed }\end{array}$ & $495(66.5)$ & $2.11 \pm 2.16$ \\
\hline Changed (33.5\%) & It has changed. & $249(33.5)$ & $2.30 \pm 2.34$ \\
\hline \multirow{3}{*}{$\begin{array}{l}\text { If changed, } \\
\text { please state the } \\
\text { best describes } \\
\text { your opinion } \\
\text { about the effect } \\
\text { of pandemic on } \\
\text { your disease? }\end{array}$} & $\begin{array}{l}\text { I care more about } \\
\text { not getting } \\
\text { infected with the } \\
\text { virus. }\end{array}$ & $81(10.9)$ & \\
\hline & $\begin{array}{l}\text { If I get infected } \\
\text { with the virus, } \\
\text { MS symptoms } \\
\text { will get worse. }\end{array}$ & $158(21.2)$ & \\
\hline & $\begin{array}{l}\text { The stress } \\
\text { caused by the } \\
\text { pandemic causes } \\
\text { an increase in MS } \\
\text { complaints. }\end{array}$ & $10(1.3)$ & \\
\hline${ }^{*} p<0.05$ & & & \\
\hline
\end{tabular}

EDSS: Expanded Disability Status Scale, MS: Multiple sclerosis

reasons, including patients' fear of going out, inability to reach the clinic, and travel restrictions (Table 6).

One hundred eighty-six respondents (25\%) reported less physical activity, 127 (17.1\%) more physical activity, 122 (16.5\%) continued physical activity as usual. Three hundred eight (41.4\%) reported that they did not perform any physical activity in the past and did not do so during the pandemic (Table 7).

\section{Discussion}

This study investigated the changes in the attitudes towards MS due to the pandemic, the frequency of going out, the rate of treatment and routine examination interruption, and physical activity behaviors. We also revealed the characteristics of a large sample during the COVID-19 pandemic. Our results showed 


\begin{tabular}{|c|c|c|c|}
\hline & Given answers & n (\%) & EDSS \\
\hline \multirow{2}{*}{$\begin{array}{l}\text { Please state the best option to describe your opinion about } \\
\text { the frequency of going out during the pandemic. }\end{array}$} & Not changed & $114(15.3)$ & $1.16 \pm 1.57^{\mathrm{a}}$ \\
\hline & Never get out & $129(17.3)$ & $4.26 \pm 2.58^{c}$ \\
\hline \multirow{2}{*}{$\begin{array}{l}\text { Please state the best option to describe your opinion about } \\
\text { the effect of staying at home during the pandemic on your } \\
\text { disease. }\end{array}$} & Positive & $114(15.3)$ & $1.80 \pm 1.96^{d}$ \\
\hline & Neutral & 381 (51.2) & $2.40 \pm 2.49^{f}$ \\
\hline
\end{tabular}

EDSS: Expanded Disability Status Scale, MS: Multiple sclerosis

${ }^{a}$ Not changed vs. never get out $p<0.001,{ }^{b}$ The decreased frequency vs. not changed $p<0.001$, Never get out vs. the decreased frequency $p<0.001,{ }^{d}$ Positive vs. Neutral $p=0.028$, eNegative vs. positive $p=0.812,{ }^{\text {'N }}$ egative vs. neutral $p=0.072$

\section{Table 5. The rate of treatment interruption}

\begin{tabular}{|c|c|c|}
\hline & Given answers & n $(\%)$ \\
\hline \multirow{6}{*}{$\begin{array}{l}\text { Is there any interruption in } \\
\text { your treatment due to the } \\
\text { pandemic? }\end{array}$} & Not changed & $699(94.0)$ \\
\hline & I cannot get my drugs from pharmacies. & $2(0.3)$ \\
\hline & I could not get drugs because I was afraid to go out. & $3(0.4)$ \\
\hline & I could not get monthly methylprednisolone because I could not go to the hospital. & $25(3.4)$ \\
\hline & I quit treatment because I am afraid of getting COVID-19. & $6(0.8)$ \\
\hline & Pregnancy & $9(1.2)$ \\
\hline
\end{tabular}

COVID-19: Coronavirus disease-2019

Table 6. The rate of interruption in routine examination

\begin{tabular}{|l|l|l|}
\hline & Given answers & n (\%) \\
\hline \multirow{4}{*}{$\begin{array}{l}\text { Is there any interruption in your } \\
\text { routine examination due to the } \\
\text { pandemic? }\end{array}$} & There is no interruption in my planned appointment. & 446 (59.9) \\
\cline { 2 - 3 } & $\begin{array}{l}\text { My planned appointment has been canceled, but my examination has been performed } \\
\text { via phone by neurologist. }\end{array}$ & 242 (32.5) \\
\cline { 2 - 4 } & I could not my control appointment because I'm afraid to go out. & 30 (4.0) \\
\cline { 2 - 4 } & My planned appointment has been canceled / I cannot reach the clinic. & 13 (1.7) \\
\cline { 2 - 3 } & I could not my control appointment due to travel restrictions. & 13.7) \\
\hline
\end{tabular}

\section{Table 7. Physical activity behavior during the pandemic}

\begin{tabular}{|l|l|l|}
\hline & Given answers & n (\%) \\
\hline \multirow{4}{*}{$\begin{array}{l}\text { Please state the option that best } \\
\text { describes your physical activity } \\
\text { behavior during the pandemic. }\end{array}$} & $\begin{array}{l}\text { I have not performed any physical activity in the past and did not do so during } \\
\text { the pandemic. }\end{array}$ & 308 (41.4) \\
\cline { 2 - 3 } & I performed less physical activity during the pandemic. & $186(25.0)$ \\
\cline { 2 - 4 } & I continued performing my physical activity as usual during the pandemic. & 122 (16.5) \\
\cline { 2 - 4 } & I performed more physical activity than usual during the pandemic. & 127 (17.1) \\
\hline
\end{tabular}

that most pwMS did not change their attitudes towards the disease. Although the rate of going out and physical activity level decreased, there was no interruption in their medical treatments, and routine examinations were continued by phone.

Most of the pwMS have made an effort to adopt stringent preventive measures during the pandemic. A great deal of states, such as home confinement, losing social support, feelings of hopelessness, deprivation, and dispiritedness, seem cause to precipitate the depression and anxiety symptoms (7). The personal opinions on chronic disease and the illness perception and treatment adherence can be altered in stressful pandemic situations. PwMS may make different sense of their disease and experiences during the pandemic. In a recent study, 69.9\% 
of patients have reported that they are concerned that MS diagnosis alone puts them at higher risk for COVID-19 infection (8). In our study, the majority of pwMS (66.5\%) reported that their attitudes towards the disease did not change. Although there is a lot of concern about infection in the first months of the pandemic, these results show that an early attempt by health professionals and informing the patients could help manage the distress. The fact that the rates of treatment and routine examination interruptions are very low compared to the literature also supports this result (8).

In the early months of the pandemic, there was uncertainty about whether DMTs increase the risk of infection with COVID-19 or the severity of disease in MS (9). Lately, it has been understood that the risk of acquiring the infectious disease in pwMS is similar to that in the general or disease population as long as they care social distancing, follow the recommended hygiene rules and the national health authority guidance's protocol on quarantine, and adopt the curfew isolation (10). $67.3 \%$ of responders have chosen to decrease the frequency of going out during the pandemic. Those not going out because of MS already had a higher disease duration and disability level than other pwMS (Table 3).

Previous studies asserted that social distancing and quarantine could cause limitations in receiving treatments and health service support, therefore cause feeling apprehension regarding their DMTs and exacerbating disease activity (11). This study reported no alteration in $94.0 \%$ of patients'treatment programs during the pandemic, and $59.9 \%$ of pwMS continued their routine controls. Hence, their treatment adherence seems to be relatively high despite the unprecedented conditions of the pandemic. The rate of changing the therapy programs and delaying in infusions, disruption to rehabilitative services was lower than in a similar survey study (12). These results may be due to the fact that $72.7 \%$ responders had high and upper education level in our study, and also the routine assessments were performed by health professionals with a timely teleassessment during the recruitment period. These findings can be interpreted as a significant concordance between physician-patient to enhance treatment adherence (13). Considering the role of the immune mechanisms against infection in MS, the pandemic virus may exacerbate MS disease activity irrespective of the fact that immunosuppressive/immunomodulatory therapies may theoretically increase the susceptibility to COVID-19 (14). Only three pwMS (0.4\%) discontinued immunotherapy without consultation because DMTs deteriorated the immune system. Specialists have conveyed these patients to be pay attention to potential risks (prognostic factors, rebound, recurrence, etc.) with postponement DMTs in MS and appropriately informed.

PwMS had to bear psychological impacts, stress, loneliness, cabin fever, depression, healthy anxiety, and isolation caused by the outbreak, and this period may yield them a changed lifestyle and reduced physical activity (15). Our findings showed that $25 \%$ of pwMS did less exercise than before. These results were consistent with those reporting physical activity levels in the Israeli cohort (16). However, while the rate of patients who did not exercise both before and during the pandemic was $41.4 \%$ in our sample, this rate was $10.8 \%$ in Israel. This result shows that our sample was also physically inactive before the pandemic. Surprisingly, it was observed that $33.6 \%$ of patients increased or maintained the physical activity level during the pandemic. We realized during the interviews that participants generally considered that the number of steps was adequate in their daily life (walking in the workplace, going shopping, meeting friends etc.). However, they acquired regular physical activities to report considering they adopted the curfew, quarantine, or self-isolation at home. This result can also be explained by the increased awareness of patients about physical activity, social media support, and promising use of technology (17).

\section{Study Limitations}

Several limitations were present in this study. Although our sample is quite large compared to the studies in the literature, obtaining data from a single center may affect the generalizability of the results. There may be positive cases among the patients we could not be reached. The timing of the study might also have altered the results, as participants may have reacted differently earlier or later in the ongoing pandemic. Additionally, we have not asked the type of exercises that patients performed. Longitudinal follow-up studies are needed to examine whether these effects are sustained.

\section{Conclusion}

This study showed the importance of closer monitoring and advice on risk diminishment strategies meticulously during the pandemic. Additionally, the importance of decision-making between the provider and the patient has been underlined on continuing therapy. In our study, the attitudes of the majority of pwMS towards their disease have not changed. Informing the patient (advanced age, comorbidity, use of immunosuppressive drugs, etc.) and performing examinations via teleassessment may have reduced the health anxiety of the patients and ensured that their attitudes towards the disease did not change. Furthermore, it may have contributed to keeping the adherence rate to treatment at a high level. Our study has suggested that priority should be given to increasing or maintaining physical activity and preventing sedentary behavior during the ongoing pandemic and before the pandemic.

\section{Ethics}

Ethics Committee Approval: The Noninvasive Research Ethics Board of Dokuz Eylul University approved the study protocol (decision no: 2020/15-32, date: 06.07.2020). 
Informed Consent: Informed consent was obtained verbally from all participants before the assessment during the pandemic.

Peer-review: Externally and internally peer-reviewed.

\section{Authorship Contributions}

Surgical and Medical Practices: E.K., Concept: P.Y., E.K., Design: P.Y., E.K., O.S., Data Collection or Processing: P.Y., Z.A., O.S., Analysis or Interpretation: P.Y., E.K., Z.A., Literature Search: P.Y., E.K., Z.A., O.S., Writing: P.Y., E.K., Z.A.

Conflict of Interest: Authors have nothing to disclose, neither financial interests nor non-financial interests.

Financial Disclosure: This research did not receive any specific grant from funding agencies in the public, commercial, or notfor-profit sectors.

\section{References}

1. Li LQ, Huang T, Wang YQ, Wang ZP, Liang Y, Huang TB, Zhang HY, Sun W, Wang Y. COVID-19 patients' clinical characteristics, discharge rate, and fatality rate of meta-analysis. J Med Virol 2020;92:577-583.

2. Brownlee W, Bourdette D, Broadley S, Killestein J, Ciccarelli O. Treating multiple sclerosis and neuromyelitis optica spectrum disorder during the COVID-19 pandemic. Neurology 2020;94:949-952.

3. Möhn N, Pul R, Kleinschnitz C, Prüss H, Witte T, Stangel M, Skripuletz T. Implications of COVID-19 outbreak on immune therapies in multiple sclerosis patients--lessons learned from SARS and MERS. Front Immunol 2020;11:1059.

4. Ricardo A, Carnero Contentti E, Anabel SB, Adrian LP, Orlando G, Fernando $H$, Víctor R, Fernando G, Ignacio RJ. Decision-making on management of ms and nmosd patients during the COVID-19 pandemic: A latin american survey. Mult Scler Relat Disord 2020;44:102310.

5. Giovannoni G, Hawkes C, Lechner-Scott J, Levy M, Waubant E, Gold J. The COVID-19 pandemic and :he use of MS disease-modifying therapies. Mult Scler Relat Disord 2020;39:102073.

6. Thompson AJ, Banwell BL, Barkhof F, Carroll WM, Coetzee T, Comi G, Correale J, Fazekas F, Filippi M, Freedman MS, Fujihara K, Galetta SL, Hartung HP, Kappos L, Lublin FD, Marrie RA, Miller AE, Miller DH, Montalban X, Mowry EM, Sorensen PS, Tintoré M, Traboulsee AL, Trojano M, Uitdehaag BMJ, Vukusic S, Waubant E, Weinshenker BG, Reingold SC, Cohen JA. Diagnosis of multiple sclerosis: 2017 revisions of the McDonald criteria. Lancet Neurol 2018;17:162-173

7. Shader RI. COVID-19, interferons, and depression: a commentary. Psychiatry Res 2020;291:113198.

8. Zhang Y, Staker E, Cutter G, Krieger S, Miller AE. Perceptions of risk and adherence to care in MS patients during the COVID-19 pandemic: A crosssectional study. Mult Scler Relat Disord 2021;50:102856.

9. Ritchie Al, Singanayagam A. Immunosuppression for hyperinflammation in COVID-19: a double-edged sword? Lancet 2020;395:1111.

10. Parrotta E, Kister I, Charvet L, Sammarco C, Saha V, Charlson RE, Howard J, Gutman JM, Gottesman M, Abou-Fayssal N, Wolintz R, Keilson M, Fernandez-Carbonell C, Krupp LB, Zhovtis Ryerson L. COVID-19 outcomes in MS: Observational study of early experience from NYU Multiple Sclerosis Comprehensive Care Center. Neurol Neuroimmunol Neuroinflamm 2020;7:835

11. Potagas C, Mitsonis C, Watier L, Dellatolas G, Retziou A, Mitropoulos P, Sfagos C, Vassilopoulos D. Influence of anxiety and reported stressful life events on relapses in multiple sclerosis: a prospective study. Mult Scler 2008;14:12621268.

12. Moss BP, Mahajan KR, Bermel RA, Hellisz K, Hua LH, Hudec T, Husak $S$, McGinley MP, Ontaneda D, Wang Z, Weber M, Tagliani P, Cárdenas-Robledo S, Zabalza A, Arrambide G, Carbonell-Mirabent P, Rodríguez-Barranco M, Sastre-Garriga J, Tintore M, Montalban X, Douglas M, Ogbuokiri E, Aravidis B, Cohen JA, Mowry EM, Fitzgerald KC. Multiple sclerosis management during the COVID-19 pandemic. Mult Scler 2020;26:1163-1171.

13. Neter E, Glass-Marmor L, Haiien L, Miller A. Concordance Between Persons with Multiple Sclerosis and Treating Physician on Medication Effects and Health Status. Patient Prefer Adherence 2021;15:939-943.

14. Berger JR, Brandstadter R, Bar-Or A. COVID-19 and MS disease-modifying therapies. Neurol Neuroimmunol Neuroinflamm 2020;7:761.

15. Moccia M, Lanzillo R, Brescia Morra V, Bonavita S, Tedeschi G, Leocani L, Lavorgna L; Digital Technologies Web and Social Media Study Group of the Italian Society of Neurology. Assessing disability and relapses in multiple sclerosis on tele-neurology. J Neurol Sci 2020;41:1369-1371.

16. Kalron A, Dolev M, Greenberg-Abrahami M, Menascu S, Frid L, AvrechShezifi S, Harari G, Magalashvili D, Achiron A. Physical activity behavior in people with multiple sclerosis during the COVID-19 pandemic in Israel: Results of an online survey. Mult Scler Relat Disord 2021;47:102603.

17. Parker K, Uddin R, Ridgers ND, Brown H, Veitch J, Salmon J, Timperio A, Sahlqvist S, Cassar S, Toffoletti K, Maddison R, Arundell L. The Use of Digital Platforms for Adults' and Adolescents' Physical Activity During the COVID-19 Pandemic (Our Life at Home): Survey Study. J Med Internet Res 2021;23:e23389. 